\title{
Studies on the Influence of Dietary Cholesterol on Cholesterol Metabolism in the Isotopic Steady State in Man *
}

\author{
Jean D. Wilson † and Charles A. Lindsey, JR. \\ (From the Department of Internal Medicine, The University of Texas Southwestern Medical \\ School, Dallas, Texas)
}

The relation between dietary cholesterol and the concentration of cholesterol in serum has been the subject of intense interest since the original demonstration that both hypercholesterolemia and atherosclerosis could be induced in the rabbit by the feeding of diets rich in cholesterol (2). On the other hand, in man virtually all studies have demonstrated that rather wide variations in the cholesterol intake result in less marked effects on the serum cholesterol (3-8). Even when the intake of dietary cholesterol greatly exceeds the normal turnover rate, the concentration of cholesterol in the serum almost never rises more than $100 \mathrm{mg}$ per $100 \mathrm{ml}(8)$, and indeed, in careful balance studies it is necessary to compare cholesterol diets with diets totally devoid of cholesterol before any significant changes can be detected (4-7). This ability of the serum cholesterol of man to stabilize at only moderately elevated levels despite enormous intake resembles the situation in such animals as the rat (9) and is in marked contrast to the profound degrees of hypercholesterolemia produced in the rabbit by cholesterol feeding (2).

At least three compensatory mechanisms could account for this phenomenon. First, dietary cholesterol suppresses the hepatic synthesis of cholesterol in the human (10) as well as in other species (11) via a negative feedback system. However, the suppression of hepatic synthesis cannot be the only compensatory mechanism since

* Submitted for publication April 29, 1965; accepted July 15, 1965 .

Supported by grants from the U. S. Public Health Service and the American Heart Association.

The results have been published in abstract form (1).

$\dagger$ Work performed during tenure as an Established Investigator of the American Heart Association.

Address requests for reprints to Dr. Jean D. Wilson, Dept. of Internal Medicine, The University of Texas Southwestern Medical School, Dallas, Texas 75235. it can quantitatively account only for a degree of protection equal to that amount ordinarily synthesized in the liver, whereas the serum cholesterol remains virtually stable despite the ingestion of quantities of cholesterol many times this value. Second, the ingestion of cholesterol could cause an increase in the rate of degradation of cholesterol to bile acids. This phenomenon has been clearly documented in the rat $(12,13)$; in this species increased bile acid production occurring with very effective cholesterol absorption is the major means by which serum cholesterol is stabilized. Finally, it is obvious that any effect of exogenous cholesterol on cholesterol metabolism is dependent on its rate of absorption. Because cholesterol is both absorbed and secreted across the wall of the intestine, the best means of quantifying cholesterol absorption in the intact animal involves the use of a complicated double isotopic steady state technique (13), and, consequently, very little is known about net cholesterol absorption in various species.

To evaluate in the human the possible roles of these three factors-the inhibition of cholesterol synthesis, increased bile acid production, and a limited ability to absorb dietary cholesterol-five balance studies have been performed in two normal men who were fed varying quantities and types of cholesterol-4-14 $\mathrm{C}$ until an isotopic steady state was attained. The results clearly demonstrate that dietary cholesterol, whether fed in the form of eggs or in a purified state, has little effect on the total rate of endogenous cholesterol synthesis or of cholesterol degradation to bile acids. We have concluded, therefore, that in man the major mechanism that stabilizes the serum cholesterol during large variations in the intake of exogenous cholesterol is a limited capacity for cholesterol absorption. 


\section{Methods}

Two normal men, ages 31 and 23 , were studied during five different feeding periods varying from 21 to 40 days over an 8-month interval. During the periods of study the subjects were ambulatory outpatients who ate only a constant weighed quantity of formula diet, vitamins, Hawk-Oser salt mix, and clear liquids. After a steady state had been approached, stools were collected into refrigerated, tared aluminum containers for periods of time that varied from 4 to 7 days; the stool samples. were pooled and treated as a single sample for the purpose of the subsequent analyses.

Two different formula diets were utilized in these studies. For the periods in which crystalline cholesterol was added to the diets the basic formulas consisted of triolein, ${ }^{1}$ vitamin-free casein, ${ }^{1}$ gelatin, sucrose, vanilla extract, egg white, and methyl cellulose; the analyzed sterol content of this basic formula was less than $5 \mathrm{mg}$ of digitonin precipitable material per day. Varying amounts of crystalline cholesterol-4- ${ }^{14} \mathrm{C}$ were added to this basic diet by dissolving the cholesterol in a portion of the triolein and feeding it directly in either two or three portions per day. The cholesterol-4- ${ }^{14} \mathrm{C}$ used in these studies was obtained by adding cholesterol-4- ${ }^{14} \mathrm{C}^{2}$ that had been purified by chromatography on silica gel in benzene : ethyl acetate (4:1) (14) to cholesterol that had been regenerated from the dibromide and then recrystallized five times (15). This mixture was then recrystallized three times from ethanol.

For the formula diets in which the cholesterol source was egg yolk, the eggs were obtained as follows: eight laying hens were each injected with $1.2 \times 10^{8} \mathrm{cpm}$ of cholesterol-4- ${ }^{14} \mathrm{C}$ dissolved in ethanol, and all the eggs were collected for 1 month. The pooled, frozen yolks (6 dozen) were then diluted with 140 dozen fresh egg yolks. This mixture was stirred repeatedly in two large batches and frozen in weighed aliquots. The formula for this diet consisted of sucrose, vanilla extract, gelatin, and egg yolk that were whipped together and then frozen. The total daily caloric intakes for each of the study periods and the calculated distribution of the calories are given in Table I. In each instance the cholesterol content and the cholesterol specific activity were measured directly by analysis of the entire diet.

At the end of the first feeding period a study of the change in specific activity of the dietary cholesterol at various levels in the gastrointestinal tract was performed in each of the subjects. A Levine tube was. allowed to pass overnight into the duodenum $(95 \mathrm{~cm}$ from the mouth), and then a second tube was passed into the stomach (65 cm from the mouth). Fasting samples of gastric juice and succus entericus mixed with bile were then aspirated, and during the next 24 hours the tubes were allowed to pass to the jejunum $(150 \mathrm{~cm})$ and the ileum $(250 \mathrm{~cm})$, respectively, and fasting samples were again collected the next morning. In each instance the

\footnotetext{
1 Nutritional Biochemicals, Cleveland, Ohio.
}

2 New England Nuclear Corp., Boston, Mass. position of the tip of the tube as evidenced by a mercury marker was checked by fluoroscopy just before the collection of the samples, and consequently, although telescoping of bowel around the tubes can occur, the positioning of the tubes was thought to be reliable.

Serum cholesterol analysis. The serum cholesterol specific activity and content were measured as follows: $2 \mathrm{ml}$ of serum was mixed with $0.4 \mathrm{ml}$ of $10 \mathrm{~N} \mathrm{KOH}$ and saponified in an autoclave for 30 minutes at 15 pounds pressure. An equal volume of ethanol was then added, and the solution was brought to a boil on a steam bath. Neutral sterols were then extracted into a tenfold excess of ethyl ether, and after backwashing the ether extracts were dried. After dissolving the residue in acetone-alcohol $(1: 1)$ cholesterol digitonides were then formed, washed, and dried by the method of Sperry and Webb (16). The cholesterol digitonides were dissolved in methanol; one aliquot was added to $0.4 \%$ diphenyloxazole in toluene and assayed for ${ }^{10} \mathrm{C}$ in a Packard liquid scintillation spectrometer, and one aliquot was assayed for cholesterol content (16). Each determination was performed in duplicate. Specific activity determinations performed in this way varied less than $5 \%$ when rechecked on different days.

Bile acid and neutral sterol content of feces. To the weighed feces samples was added 2 vol of water, and the mixture was shaken on a paint can shaker for 5 minutes and then transferred to a Waring blendor and homogenized for 5 minutes. One- to 2-g samples of the liquid mixture were then carefully weighed in triplicate, mixed with 100 $\mathrm{ml}$ chloroform: methanol $(2: 1)$, and refluxed for $5 \mathrm{~min}$ utes. The chloroform: methanol extracts were filtered by washing into $250-\mathrm{ml}$ Erlenmeyer flasks, dried under nitrogen by boiling, mixed with $3.5 \mathrm{ml}$ of $10 \mathrm{~N} \mathrm{NaOH}$ and $10 \mathrm{ml} \mathrm{H} \mathrm{H}_{2} \mathrm{O}$, and autoclaved for 3 hours at 15 pounds pressure and $130^{\circ} \mathrm{C}$. Two vol of ethanol was then added, and the samples were refluxed on a hot plate for $5 \mathrm{~min}$ utes. Neutral sterols were extracted three times from the mixture by shaking with pentane; the pentane extracts were combined, backwashed with $10 \mathrm{ml}$ of ethanol: water $(1: 1)$, and dried under nitrogen. After dissolving the residue in methanol one aliquot was assayed for ${ }^{14} \mathrm{C}$, and one was saved for thin-layer chromatography. The water layer remaining after the pentane extraction was acidified to $\mathrm{pH} 2$ to 3 by titrating with concentrated $\mathrm{H}_{2} \mathrm{SO}_{4}$, and the bile acid fraction was extracted into ethyl ether. The ethyl ether extracts were dried under nitrogen and dissolved in $50 \mathrm{ml}$ acetone. The acetone extract was then passed through a Florisil column $(1 \times 10 \mathrm{~cm})$, and the bile acids were eluted with $50 \mathrm{ml}$ of acetone: methanol: acetic acid $(80: 20: 1)(17)$. The eluate was then dried on a steam bath, and the residue was dissolved in methanol. One aliquot was assayed for ${ }^{14} \mathrm{C}$, and one was chromatographed by thin-layer chromatography. In every instance the samples were reassayed for ${ }^{14} \mathrm{C}$ after the addition of an internal standard, and appropriate corrections for quenching were made.

To separate cholesterol from bacterial transformation products, the neutral sterol fractions were chromatographed on silica gel plates in benzene: ethyl acetate 
DIETARY CHOLESTEROL IN MAN

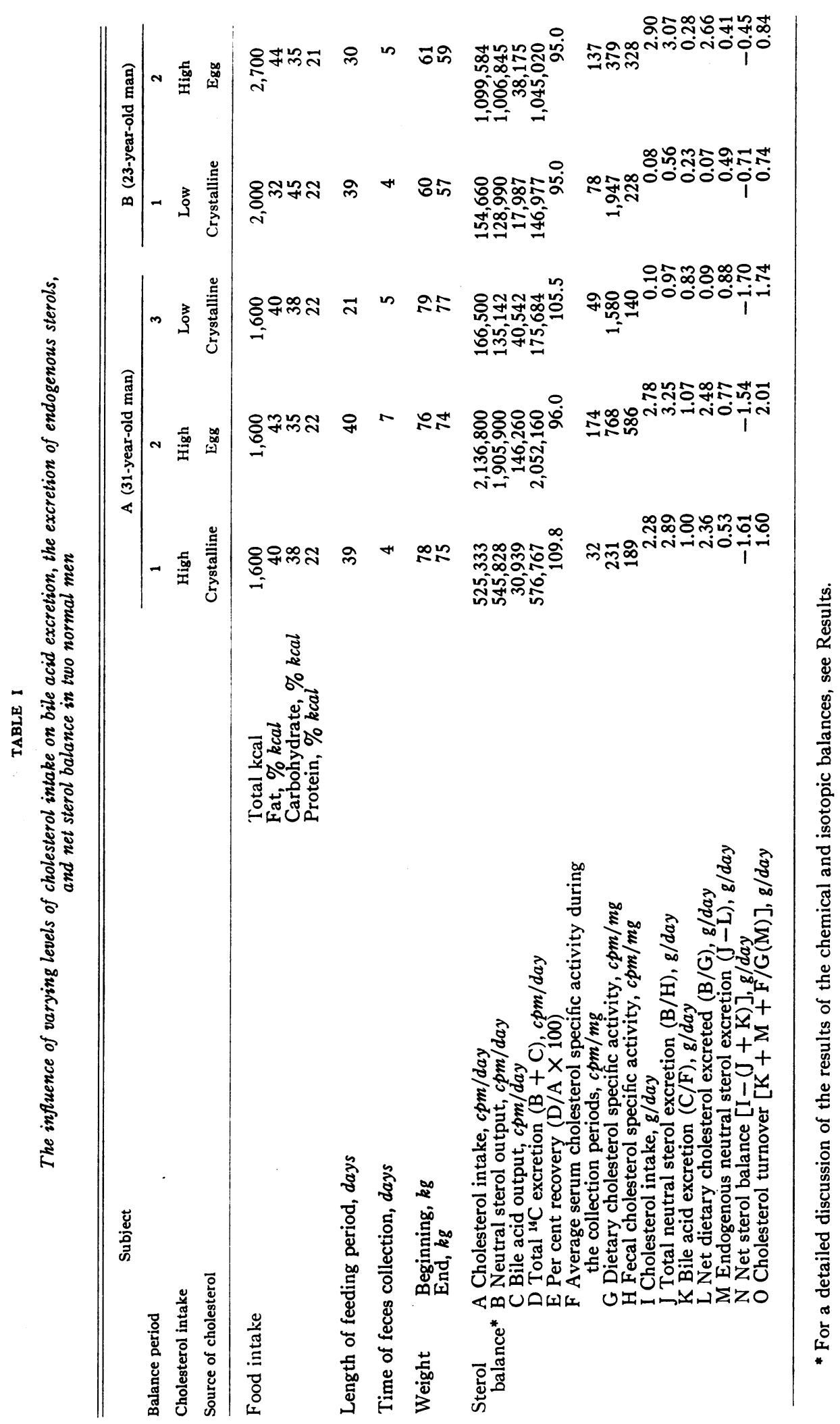




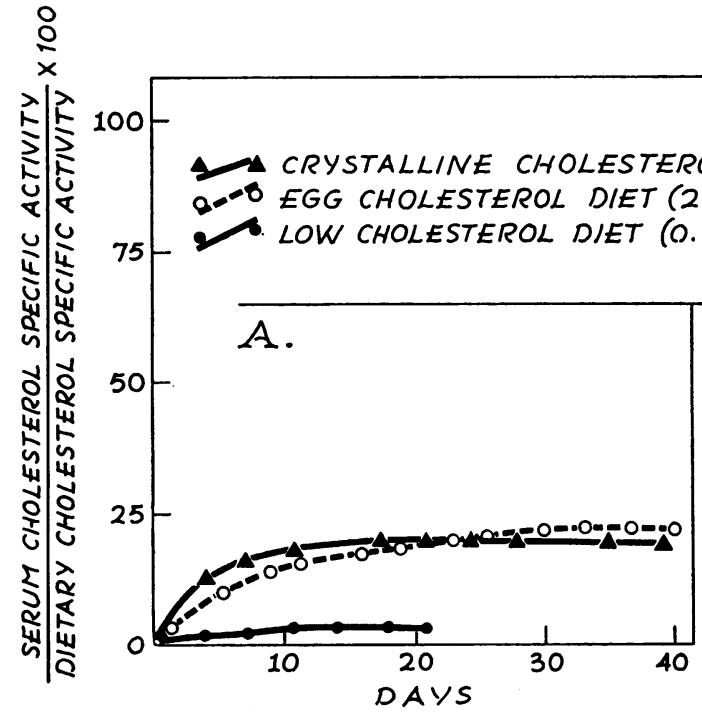

Fig. 1. The influence of high and Low Cholesterol- $-4-{ }^{14}$ C diets on the SPECIFIC ACtivity (A) AND the CONCENTRATion (B) of SERUm Cholesterol in Subject A.

(2:1) (14), and after spraying with Rhodamine $G$ the cholesterol area was scraped and eluted into acetone: ethanol $(1: 2)$. Cholesterol digitonides were formed and assayed for ${ }^{14} \mathrm{C}$ and chemical content as before. To insure that each bile acid fraction was free of neutral sterol the ethyl ether extracts were also chromatographed on silica gel plates in benzene: ethyl acetate $(4: 1)$ and sprayed with Rhodamine $G$; in this system the bile acids remained near the origin, and any contaminating cholesterol or coprostanol moved near the front; in some instances a small amount of radioactivity could be located in the cholesterol and coprostanol areas, and consequently the necessary corrections were made. With this system the recovery of cholesterol-4- ${ }^{14} \mathrm{C}$ and cholic acid-4- ${ }^{11} \mathrm{C}$ added to feces samples was checked; the recoveries averaged $91 \%$ for cholesterol and $96 \%$ for cholic acid.

Analysis of diet and succus entericus. The weighed formula intake along with the daily portions of salt mix, clear liquids, vitamins, and methyl cellulose were homogenized in a Waring Blendor in $2 \mathrm{~L}$ of chloroform : methanol $(2: 1)$. The mixture was filtered, and $5-\mathrm{ml}$ aliquots in triplicate were dried and assayed as described for the stool samples for cholesterol content and ${ }^{14} \mathrm{C}$. The aspirates from the gastrointestinal tract (averaging $10 \mathrm{ml}$

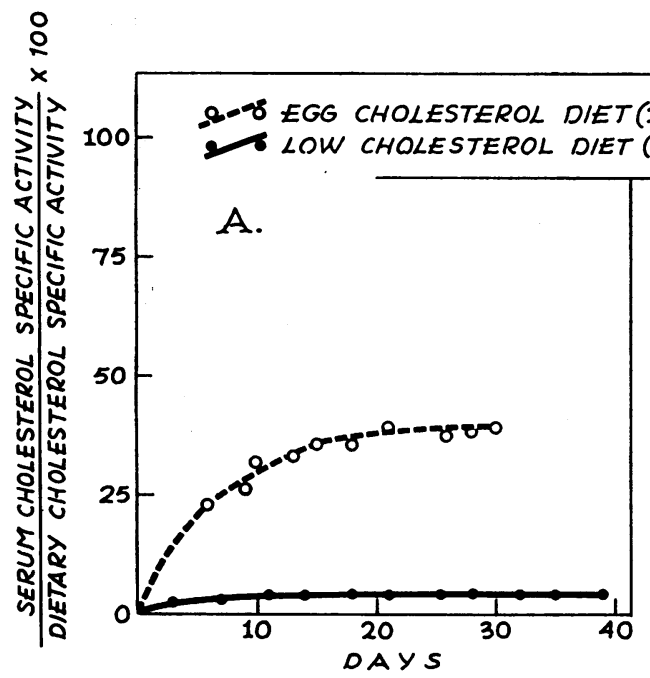

Fig. 2. The influence of High and LOW ChOlesterol- $-4-{ }^{14} \mathrm{C}$ DIETS ON THE SPECIFIC ACtivity (A) and the concentration (B) of Serum cholesterol in Subject B. 
in volume) were mixed with $\frac{1}{10}$ vol of $10 \mathrm{~N} \mathrm{NaOH}$ and autoclaved for 3 hours at 15 pounds pressure and $250^{\circ} \mathrm{C}$. An equal volume of ethanol was then added, and neutral sterols were extracted with pentane and chromatographed on plates of silica gel. The cholesterol areas were scraped and eluted, and the digitonides were assayed for ${ }^{14} \mathrm{C}$ and cholesterol content as before.

\section{Results}

The influence of feeding two normal subjects diets containing varying amounts of cholesterol${ }^{4-14} \mathrm{C}$ on the specific activity and the content of serum cholesterol is illustrated in Figures 1 and 2. In each instance, the specific activity of the dietary cholesterol was directly measured by analysis of the total daily dietary intake during the study. The lengths of the five feeding periods for these two subjects varied from 21 to 40 days, and in each of these periods an isotopic steady state was approached by the end of the study. In the case of the low cholesterol diets ( 0.10 and $0.08 \mathrm{~g}$ per day) a steady state was attained within 10 days. When diets high in cholesterol were fed, however, an isotopic steady state was not approached until after 20 to 30 days of feeding. In Subject A who was fed high cholesterol diets in which the source was either crystalline cholesterol or egg, the ratios of the specific activity of the serum cholesterol to that of the diet in the isotopic steady state (18 and $24 \%$ ) were similar. indicating that the source of the cholesterol had little influence on the degree of equilibration achieved. The percentage of equilibration between diet and serum in these studies (2.5 and $4 \%$ for the low cholesterol diets and 18, 24, and $38 \%$ for the high cholesterol diets) is similar to the results obtained by Taylor, Patton, Yogi, and Cox (18), and by Kaplan, Cox, and Taylor (19). This confirmation is striking in view of the fact that in the latter studies the specific activity of dietary cholesterol was a calculated and not a directly determined value. The results mean that even when the ingested cholesterol grossly exceeds endogenous production, no more than 25 to $40 \%$ of the serum cholesterol in these two subjects is derived from the diet, regardless of whether the source is cholesterol in a naturally occurring (egg) or purified form.

Furthermore, the falls in serum cholesterol (Figures 1 and 2, sections B) in these two sub- jects after the feeding of semisynthetic diets very low in cholesterol content (20 and $35 \mathrm{mg}$ per $100 \mathrm{ml}$ ) are very similar to the results observed by Conner, Hodges, and Bleiler (5). In these two subjects the differences between several weeks of feeding diets containing either very low or very high concentrations of cholesterol were 50 and $55 \mathrm{mg}$ per $100 \mathrm{ml}$ in the case of Subject A and $50 \mathrm{mg}$ per $100 \mathrm{ml}$ in the case of Subject B. It can be concluded, therefore, that these two subjects exhibited a typical response of serum cholesterol concentration to variations in cholesterol ingestion.

The results of the balance data obtained from the five study periods in these subjects are illustrated in Table I. Because of unpalatability, the caloric intake of these semisynthetic diets was only 1,600 to $2,700 \mathrm{kcal}$ per day. Nevertheless, as illustrated by the weights, the maximal weight change was only $3 \mathrm{~kg}$ for a 39-day study period, and in each instance the major weight change occurred during the first 2 weeks of the study. Under the section labeled sterol balance are listed the results of the chemical and isotopic balances. The cholesterol intake and the neutral sterol, bile acid, and total ${ }^{14} \mathrm{C}$ excretion each day are listed in columns A, B, C, and D. The per cent recovery of the ingested radioactive cholesterol is listed in column $\mathrm{E}$; it can be seen that the recovery varied from 95 to $110 \%$, and consequently it can be concluded that errors in the subsequent calculations due to imperfections in collection technique cannot be more than $10 \%$. In these studies the total neutral sterol excretion, representing the sum of cholesterol and coprostanol, was determined as follows: total neutral sterol excretion (grams per day) = neutral sterol output (counts per minute per day)/fecal cholesterol specific activity (counts per minute per milligram) $\times 1,000$. The results of these determinations are listed in column J. As would be expected, total neutral sterol excretion closely paralleled cholesterol intake, but in each of the five studies total excretion slightly exceeded the intake. Bile acid excretion in the isotopic steady state can be calculated as follows: bile acid excretion (grams per day) ${ }^{3}=$ bile acid output (counts per minute

${ }^{8}$ Since the molecular weights of cholesterol and bile acid are similar but not identical, this formulation should only 
per day)/average serum cholesterol specific activity (counts per minute per milligram) $\times 1,000$. The bile acid excretions for these balances are listed in column $\mathrm{K}$. The bile acid excretion in these two subjects was markedly different; it averaged $0.26 \mathrm{~g}$ per day in Subject B and 0.97 $\mathrm{g}$ per day in Subject A. However, it is quite clear that in these subjects bile acid excretion was surprisingly constant regardless of the type or quantity of dietary cholesterol; under circumstances in which dietary cholesterol was increased 30 -fold, bile acid excretion was 0.83 and $1.07 \mathrm{~g}$ per day in Subject $A$ and 0.23 and $0.28 \mathrm{~g}$ per day in Subject B. Within the limits of this methodology it is very likely that these slight changes are not significant. Furthermore, it is certain that even if such small changes are real, increased bile acid excretion cannot be the major protective device since they could only account for a trivial portion of the ingested cholesterol.

Several other calculations were made from these data. The net dietary cholesterol excreted (column L) was determined as follows: net dietary cholesterol excreted (grams per day) = neutral sterol output (counts per minute per day)/dietary cholesterol specific activity (counts per minute per milligram) $\times 1,000$. This value represents the portion of the fecal neutral sterol that was derived ultimately from the diet; it is a net figure and does not indicate whether the cholesterol passed through the gastrointestinal tract unabsorbed or was absorbed and re-excreted. As would be predicted in the isotopic steady state this value in each instance closely parallels the intake. It is also possible to determine the endogenous neutral sterol excretion under these conditions (column $\mathrm{M}$ ): endogenous neutral sterol excretion (grams per day) $=$ total neutral sterol excretion (grams per day) - net dietary cholesterol excreted (grams per day). This value represents the amount of cholesterol synthesized and excreted as neutral sterol from the body each day; it is not the same as the total amount synthesized per day since a portion of the total is catabolized to bile acids. However, it does represent the total endogenous contribution to one of

be regarded as a close approximation; e.g., the molecular weights of cholesterol, deoxycholic acid, and cholic acid are 387,392 , and 409. the two pathways of cholesterol elimination from the body. It is striking that when cholesterol was fed in large quantities the endogenous sterol fell only from 0.88 to 0.53 and $0.77 \mathrm{~g}$ per day in Subject A and from 0.49 to $0.41 \mathrm{~g}$ per day in Subject B. Therefore, despite the operation in man of the hepatic negative feedback (11), some cholesterol continues to be synthesized. This finding confirms results obtained by Taylor and co-workers using $\mathrm{D}_{2} \mathrm{O}(20)$ and clearly means either that the suppression of cholesterol synthesis in the liver is incomplete or that a major nonhepatic source for the synthesis of endogenous cholesterol is present in the human. This conclusion is supported by the determinations of net sterol balance (column $\mathrm{N}$ ) : net sterol balance $=$ cholesterol intake (grams per day) - [total neutral sterol excretion (grams per day) + bile acid excretion (grams per day)]. In every instance of both high and low intake the net sterol balance is negative; furthermore, the degree of negative balance is affected slightly if at all by the quantity of cholesterol ingested. It is obvious, then, that cholesterol synthesis continues during high cholesterol intake. The net sterol balance on the low cholesterol diets $(-1.70 \mathrm{~g}$ per day in Subject A and $-0.71 \mathrm{~g}$ per day in Subject B) should equal, furthermore, cholesterol turnover in the steady state. It is interesting that these values are similar to values of cholesterol turnover rate in the human reported by Chobanian, Burrows, and Hollander (21) by the analysis of disappearance curves after a single injection of cholesterol$4-{ }^{14} \mathrm{C}$. This remarkable similarity (their mean value being $1.68 \pm 0.98 \mathrm{~g}$ per day) is even more striking in view of the fact that dietary cholesterol was uncontrolled in their study and that the intake of dietary cholesterol might increase the turnover rate.

Cholesterol turnover rate can be approximated in another way from these data (column $\mathrm{O}$ ) : cholesterol turnover (grams per day) $=$ bile acid excretion (grams per day) + endogenous neutral sterol excretion (grams per day) + [percentage of serum cholesterol derived from diet $X$ endogenous neutral sterol excretion (grams per day)].

This formulation adds a correction factor for the cholesterol absorbed and re-excreted into the feces as neutral sterol in the steady state. The values obtained from this estimation agree rea- 
sonably well, in the case of the low cholesterol diets, with the estimate from the net sterol balance. In two of the three cases the estimate for turnover by this method is slightly higher in the case of the high cholesterol diets.

The calculation of cholesterol turnover on the high intake makes possible one other approximation from these balance figures. If, in the isotopic steady state the specific activity of serum cholesterol represents the specific activity of the entire miscible pool, then a maximal value for net cholesterol absorption can be determined: maximal net cholesterol absorption (grams per day) $=$ percentage of serum cholesterol derived from the diet $\times$ turnover rate of cholesterol (grams per day). It must be emphasized that this value is a maximal value for cholesterol absorption and cannot be assumed to be equal to the absolute absorption rate for two reasons: radioactive cholesterol can exchange across the gut wall without representing net movement, and cholesterol can be excreted and reabsorbed via an enterohepatic circulation. However, with these limitations in mind the value for maximal net absorption is of interest; in these two individuals the maximal absorption of dietary cholesterol was $0.32 \mathrm{~g}$ per day (Subject A) and $0.34 \mathrm{~g}$ per day (Subject B). This means that at the most only 10 to $15 \%$ of the ingested cholesterol was absorbed in this study, and it is very likely that the real net value is considerably less than this. Quite clearly, this limited ability to absorb dietary cholesterol is quantitatively the most important of the devices that protect against dietary cholesterol-induced hypercholesterolemia in the human.

To obtain some insight into the site of the equilibration of dietary cholesterol with endogenous cholesterol in the isotopic steady state, the specific activity of the cholesterol was determined at various levels of the gastrointestinal tract at the end of the first period of study in Subjects $A$ and $B$ (Figure 3 ). It is obvious that if the specific activity of the serum cholesterol never equals that of the diet in the steady state, ingested cholesterol will be diluted with endogenous cholesterol as it passes along the gastrointestinal tract. If the bile is the source for mixing endogenous and exogenous cholesterol, then a fall in specific activity of dietary cholesterol would be expected to occur in the duodenum; further-

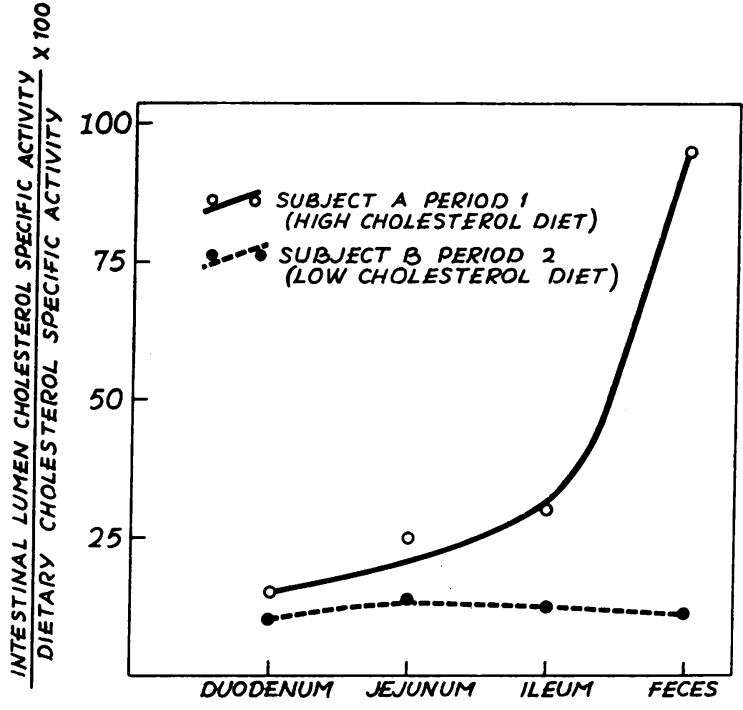

Fig. 3. The change in SPECIfIC activity of Dietary CHOLESTEROL-4- ${ }^{14} \mathrm{C}$ DURING THE POSTABSORPTIVE CONDITION AT VARIOUS LEVELS IN THE GASTROINTESTINAL TRACT IN THE ISOTOPIC STEADY STATE.

more, if, in addition, cholesterol not in equilibrium with the blood is secreted from the wall of the gastrointestinal tract, then one would expect a further fall of the specific activity of the lumenal cholesterol in the steady state at sites distal to the common bile duct. In the studies shown in Figure 3 the cholesterol content of the postabsorption gastric aspirate was too low for an accurate determination of the specific activity; at all other levels sampled in the gastrointestinal tract adequate samples were obtained for this analysis. In both studies maximal dilution of the specific activity was obtained in the duodenal aspirate, and this specific activity was in each instance almost equal to that of the serum; in the subject on the low cholesterol intake (Subject $B$ ), the specific activity remained virtually unchanged throughout the remainder of the gastrointestinal tract; in this individual cholesterol turnover ( $0.74 \mathrm{~g}$ per day) enormously exceeded cholesterol intake ( $0.08 \mathrm{~g}$ per day), and it may be concluded that complete mixing and dilution occurred by the time bile mixed with the exogenous sterol. This is certainly not surprising in view of the fact that in the human bile is poured out from the gall bladder just at the appropriate time to mix with the exogenous sources. The fact that no further dilution occurs down the 
gastrointestinal tract, furthermore, implies that if secretion of cholesterol not in equilibrium with blood occurs across the intestinal wall it must be quantitatively insignificant. The situation in the study of the specific activity along the intestine of the subject on the high cholesterol intake is slightly more complex. Again maximal dilution occurred by the time the duodenum was reached, and this specific activity was approximately that of the serum. However, in this study cholesterol intake ( $2.28 \mathrm{~g}$ per day) grossly exceeded the turnover rate $(1.60 \mathrm{~g}$ per day) and the net excretion of endogenous sterol ( $0.53 \mathrm{~g}$ per day), and down the gastrointestinal tract the specific activity rose slightly in the small intestine and then increased significantly to approximately $90 \%$ of the dietary cholesterol by the time the feces were sampled. In other words, food containing far more cholesterol than is produced each day passes through the duodenum in a wave and is diluted slightly. As it passes on through the gastrointestinal tract the area proximal to it approaches equilibrium with blood. Again, these data do not mean that secretion of cholesterol may not occur across the intestinal wall, but they do suggest that dilution occurring by the time the bile is mixed with the diet is entirely sufficient to account for the drop in the specific activity along the length of the gastrointestinal tract even in the postabsorptive state when dilution of secreted cholesterol would be expected to be minimal.

\section{Discussion}

These studies clearly demonstrate that, of the possible mechanisms that protect the human from developing extreme hypercholesterolemia during the excessive intake of dietary cholesterol, a limited ability to absorb dietary cholesterol is quantitatively more important than inhibition of hepatic cholesterol synthesis or acceleration of cholesterol degradation to bile acids. The human thus differs in this regard from the rat; in this species serum cholesterol is kept relatively stable even during high cholesterol absorption by increasing bile acid output (13). Furthermore, although quantitative estimates of cholesterol absorption rates cannot be determined from the studies reported here, maximal values for net absorption rates were estimated to approximate about $0.3 \mathrm{~g}$ per day when $3 \mathrm{~g}$ of cholesterol was fed. These results are similar to estimates obtained for maximal cholesterol absorption ( 0.3 to $0.5 \mathrm{~g}$ per day) by another application of the steady state technique (19) and differ markedly from earlier estimates of this capacity at 1 to $3 \mathrm{~g}$ per day (2224). The actual direct estimation of this capacity in an intact organism can best be made by use of a double isotopic steady state (13).

Although these results have been interpreted as constituting strong evidence that a limitation in the ability to absorb cholesterol is the major means by which the human is protected from the development of hypercholesterolemia during high cholesterol intake, they clearly do not mean that either an inhibition of cholesterol synthesis or an increase in bile acid excretion may not be of importance in the steady state. If the human does indeed absorb $300 \mathrm{mg}$ or less of cholesterol per day then, eventually, hypercholesterolemia would result unless one or both of these mechanisms were operative. The suppression of hepatic cholesterol synthesis by the feeding of exogenous cholesterol to man (10) is entirely adequate to compensate for this quantity of cholesterol absorption; whether increased bile acid formation actually occurs as a second compensatory mechanism cannot be determined at present.

The use of the isotopic steady state also permits an analysis of the biosynthetic origin of serum cholesterol in man. If the liver were, indeed, the principal source of endogenous serum cholesterol in man (25) and if the feeding of cholesterol to man causes a shutoff of hepatic cholesterol synthesis (10), then in the steady state the specific activity of serum should approach that of the diet even when small net amounts of cholesterol are absorbed. In this study as in the experiments of Kaplan, Cox, and Taylor (19) a maximum of only about $40 \%$ of the serum cholesterol was derived from dietary sources under circumstances of high cholesterol intake. Two possible explanations for this phenomenon can be offered. First, the suppression of hepatic synthesis may be incomplete in the intact animal; in view of the demonstration that in man (10), as well as in other species (11), the inhibition of cholesterol synthesis in liver by cholesterol feeding is virtually complete, this possibility seems 
unlikely. Second, it is clear that the only tissue in which the negative feedback has been proven to be operative is the liver (26), and it is possible that other tissues such as intestine (27) might contribute ultimately to the circulating pool. The results of the present study, along with the demonstration by Taylor and associates that cholesterol synthesis, as indicated by the incorporation of $\mathrm{D}_{2} \mathrm{O}$, continues in the cholesterol-fed subject (20), are compatible with either possibility.

A third implication of this study is that purified cholesterol when fed in a soluble form has the same quantitative effect on endogenous cholesterol metabolism as does a similar amount of cholesterol in a naturally occurring state such as egg. Indeed, when two separate studies were performed in the same individual similar equilibration with the serum was obtained. This finding implies that the relative inability of the human intestine to absorb cholesterol under the conditions of the present study is not due to variations in the lipoprotein form in which it is ingested. Whether in the human this absorptive ability may be influenced by variations in the type of dietary fat or carbohydrate cannot be determined from this study. However, the lack of any difference in effect on serum cholesterol level between purified and lipoprotein-bound cholesterol is hardly surprising in view of the fact that cholesterol esters are split to free cholesterol before cholesterol absorption (28). This conclusion probably only applies in the case of cholesterol that is fed in a dissolved state since cholesterol fed in a crystalline, undissolved state is absorbed very poorly in most species (12) and, in addition, this lack of any difference in effect on cholesterol metabolism may apply only to the circumstances in which formula diets are fed.

Finally, the results of this study may be interpreted as furnishing no support for the suggestion that cholesterol not in equilibrium with the blood cholesterol may be secreted into the intestinal lumen in the human as well as in the rat (13) in the isotopic steady state. The dilution of dietary cholesterol with that of bile appears to be sufficient to explain the entire excretion of endogenous sterol. It is certain that the bile is not the only endogenous source of fecal cholesterol in the human since the human with complete biliary obstruction continued to excrete endogenous cholesterol in the feces (29); however, in the normal human the cholesterol reaching the feces from the intestinal wall may be in equilibrium with that of blood.

\section{Summary}

The technique of analysis utilizing the isotopic steady state has been applied to assess the effect of varying cholesterol intakes on cholesterol synthesis, excretion and degradation, and absorption in man. Five balance studies were performed on two normal subjects who had been fed diets containing varying amounts of cholesterol-4- ${ }^{14} \mathrm{C}$ until an isotopic steady state was attained. It has been concluded from these studies that a limited ability to absorb dietary cholesterol is more important in protecting the human from hypercholesterolemia when cholesterol is fed than is either the inhibition of cholesterol synthesis or the acceleration of bile acid excretion. In addition, the biosynthetic origin of serum cholesterol has been assessed under these circumstances; approximately 60 to $80 \%$ of the serum cholesterol is derived from endogenous sources when a diet high in cholesterol is fed.

\section{Acknowledgments}

We wish to express our appreciation to Dr. John F. Fordtran for assistance in obtaining the aspirates from the gastrointestinal tract. The technical assistance of Joanne Sherwood and George T. Crowley in these studies is gratefully acknowledged.

\section{References}

1. Lindsey, C. A., Jr., and J. D. Wilson. Effect of dietary cholesterol on cholesterol metabolism in man (abstract). Clin. Res. 1965, 13, 64.

2. Anitschkow, N. Experimental arteriosclerosis in animals in Arteriosclerosis, E. V. Cowdry, Ed. New York, MacMillan, 1933, p. 271.

3. Beveridge, J. M. R., W. F. Connell, H. L. Haust, and G. A. Mayer. Dietary cholesterol and plasma cholesterol levels in man. Canad. J. Biochem. 1959, 37, 575.

4. Connor, W. E., R. E. Hodges, and R. E. Bleiler. Effect of dietary cholesterol upon serum lipids in man. J. Lab. clin. Med. 1961, 57, 331.

5. Connor, W. E., R. E. Hodges, and R. E. Bleiler. The serum lipids in men receiving high cholesterol and cholesterol-free diets. J. clin. Invest. 1961, 40, 894. 
6. Connor, W. E., D. B. Stone, and R. E. Hodges. The interrelated effects of dietary cholesterol and fat upon human serum lipid levels. J. clin. Invest. 1964, 43, 1691.

7. Erickson, B. A., R. H. Coots, F. H. Mattson, and A. M. Kligman. The effect of partial hydrogenation of dietary fats, of the ratio of polyunsaturated to saturated fatty acids, and of dietary cholesterol upon plasma lipids in man. J. clin. Invest. 1964, 43, 2017.

8. Steiner, A., E. J. Howard, and S. Akgun. Importance of dietary cholesterol in man. J. Amer. med. Ass. 1962, 181, 186.

9. Cook, R. P., and R. O. Thomson. The absorption of fat and of cholesterol in the rat, guinea-pig, and rabbit. Quart. J. exp. Physiol. 1951, 36, 61.

10. Bhattathiry, E. P. M., and M. D. Siperstein. Feedback control of cholesterol synthesis in man. J. clin. Invest. 1963, 42, 1613.

11. Siperstein, M. D., and M. J. Guest. Studies on the site of the feedback control of cholesterol synthesis. J. clin. Invest. 1960, 39, 642.

12. Wilson, J. D. Influence of dietary cholesterol on excretion of cholesterol-4-C $\mathrm{C}^{14}$ by the rat. Amer. J. Physiol. 1962, 202, 1073.

13. Wilson, J. D. The quantification of cholesterol excretion and degradation in the isotopic steady state in the rat: the influence of dietary cholesterol. J. Lipid Res. 1964, 5, 409.

14. Avigan, J., D. S. Goodman, and D. Steinberg. Thinlayer chromatography of sterols and steroids. J. Lipid Res. 1963, 4, 100.

15. Fieser, L. F., and M. Fieser. Steroids. New York, Reinhold, 1959, p. 51.

16. Sperry, W. M., and M. Webb. A revision of the Schoenheimer-Sperry method for cholesterol determination. J. biol. Chem. 1950, 187, 97.

17. Spritz, N., E. H. Ahrens, Jr., and S. Grundy. Sterol balances in man as plasma cholesterol concentrations are altered by exchange of dietary fats. $\mathrm{J}$. clin. Invest. 1965, 44, 1482.
18. Taylor, C. B., D. Patton, N. Yogi, and G. E. Cox. Diet as source of serum cholesterol in man. Proc. Soc. exp. Biol. (N. Y.) 1960, 103, 768.

19. Kaplan, J. A., G. E. Cox, and C. B. Taylor. Cholesterol metabolism in man. Arch. Path. 1963, 76, 359.

20. Taylor, C. B., B. Mikkelson, J. A. Anderson, D. T. Forman, and G. Franz. Human serum cholesterol synthesis measured with the deuterium label (abstract). J. Lab. clin. Med. 1964, 64, 1009.

21. Chobanian, A. V., B. A. Burrows, and W. Hollander. Body cholesterol metabolism in man. II. Measurement of the body cholesterol miscible pool and turnover rate. J. clin. Invest. 1962, 41, 1738.

22. Cook, R. P., D. C. Edwards, and C. Riddell. Cholesterol metabolism. Cholesterol absorption and excretion in man. Biochem. J. 1956, 62, 225.

23. Ivy, A. C., E. Karvinen, T. M. Lin, and E. R. Ivy. Some parameters of sterol metabolism in man on a sterol- and fat-free diet. J. appl. Physiol. 1957, $11,1$.

24. Karvinen, E., T. M. Lin, and A. C. Ivy. Capacity of human intestine to absorb exogenous cholesterol. J. appl. Physiol. 1957, 11, 143.

25. Hotta, S., and I. L. Chaikoff. The role of the liver in the turnover of plasma cholesterol. Arch. Biochem. 1955, 56, 28.

26. Dietschy, J. M., and M. D. Siperstein. Personal communication.

27. Lindsey, C. A., Jr., and J. D. Wilson. Evidence for a contribution by the intestinal wall to the serum cholesterol of the rat. J. Lipid Res. 1965, 6, 171.

28. Swell, L., E. C. Trout, Jr., R. Hopper, H. Field, Jr., and C. R. Treadwell. The mechanism of cholesterol absorption. Ann. N. Y. Acad. Sci. 19581959, 72, 813.

29. Cheng, S. H., and M. M. Stanley. Secretion of cholesterol by intestinal mucosa in patients with complete common bile duct obstruction. Proc. Soc. exp. Biol. (N. Y.) 1959, 101, 223. 